\title{
Presencia de Munidopsis bermudezi en el mar Caribe colombiano (Crustacea: Anomura: Munidopsidae)
}

\section{Presence of Munidopsis bermudezi in the Colombian Caribbean Sea (Crustacea: Anomura: Munidopsidae)}

\author{
William Zubiría ${ }^{1}$ Néstor Hernando Campos $^{2 *}$ y Adriana Bermúdez ${ }^{3}$
}

\begin{abstract}
RESUMEN
Durante el desarrollo del proyecto "Caracterización ambiental en el área de interés dentro del bloque Guajira Offshore \# 3, Caribe colombiano", se recolectaron ejemplares de Munidopsis bermudezi Chace, 1939. Este es el primer registro de la especie para el mar Caribe colombiano, siendo esta la especie 46 de la superfamilia Galatheoidea registrada para esta región. Es una de las especies cercanas a Munidopsis teretis Baba, 2005, del mar de Tasmania. Se diferencia por la presencia de un par de espinas gástricas conspicuas, la ausencia de un proceso agudo en la región branquial y la ausencia de una carina en la mitad posterior del caparazón. Se presentan la descripción ampliada de la especie y la información sobre su hábitat.
\end{abstract}

Palabras claves: Nuevo registro, Decapoda, Munidopsis bermudezi, Munidopsidae, Caribe colombiano.

\begin{abstract}
Specimens of Munidopsis bermudezi Chace, 1939, were collected during the Project entitled "Environmental characterization in the area of interest within the Guajira Offshore \# 3, Colombian Caribbean block". This is the first record of this species in the Colombian Caribbean Sea. This is the 46th species of the superfamily Galatheoidea registered for this region. This species is close to Munidopsis teretis Baba, 2005, which occurs in the Tasman Sea. The former differs from the latter by the presence of a pair of conspicuous gastric spines, the absence of an acute process in the branchial region and the absence of a carina on the posterior half of the carapace. A detailed description of this species and information on its habitat are presented in this paper.
\end{abstract}

Keywords: New record, Decapoda, Munidopsis bermudezi, Munidopsidae, Colombian Caribbean Sea.

\section{INTRODUCCIÓN}

El estudio del mar profundo, entendido este como el mar cuyas aguas superan los $200 \mathrm{~m}$ de profundidad (Escobar-Briones, 2014), ha permitido incrementar el conocimiento de la biodiversidad marina mundial. Actividades como la pesca, la prospección petrolera en la plataforma y en el talud continental, el interés

\footnotetext{
Programa CSC, INVEMAR, Santa Marta, willizu@hotmail.com

CECIMAR, Universidad Nacional de Colombia, Sede Caribe, Rodadero sur, Santa Marta, Colombia, nhcamposc@unal.edu.co* Programa de Biología, Facultad de Ciencias Exactas y Naturales, Universidad de Cartagena. Grupo de Hidrobiología, abermudezt@unicartagena.edu.co
}

Recibido: 25 de abril de 2016

Corregido: 28 de junio de 2016

Aceptado: 30 de junio de 2016

DOI: http://dx.doi.org/10.15359/revmar.8-2.4 
por el estudio de nuevos hábitats que se descubren y la necesidad de conocer la diversidad marina, son algunos de los factores que han contribuido al entendimiento de estos ecosistemas que ahora se ponen al alcance, gracias al adelanto de nuevas y mejores tecnologías. La investigación de estos ambientes ha permitido aumentar el conocimiento de la biología y la distribución de un buen número de organismos marinos, así como mejorar nuestro entendimiento de la diversidad. Tal es el caso de las especies de crustáceos decápodos del orden Anomura pertenecientes a la superfamilia Galatheoidea (Navas et al. 2003; Fierro et al. 2008).

En los últimos años, la sistemática de la superfamilia Galatheoidea ha sido objeto de revisión. Ahyong et al. (2010) asignaron a esta superfamilia la familia Galatheidae Samouelle, 1819, retomaron la familia Munidopsidae Whiteaves, 1874, y describieron la nueva familia Munididae, manteniendo la familia Porcellanidae Haworth, 1825, dentro de los Galatheoidea de acuerdo con los análisis sistemáticos que respaldan la monofilia del grupo. Las familias Aeglidae, Dana, 1852; Chirostylidae Ortmann, 1892 y Kiwaidae Macpherson \& Segonzac, 2005, fueron excluidas de la superfamilia (Ahyong et al. 2010).

La familia Munidopsidae Ortmann, 1898, está compuesta por cuatro géneros: Galacantha Milne-Edwards, 1880; Leiogalathea Baba, 1969; Munidopsis Whiteaves, 1874, y Shinkaia Baba \& Williams, 1998, siendo Munidopsis el género tipo de la familia. Esta familia se caracteriza porque el flagelo sobre el primer maxilípedo está ausente o es reducido, en la mayoría de casos presentan ojos reducidos y ornamentación dorsal sobre el caparazón. En el ámbito mundial, hasta el año 2008, el número de especies del género Munidopsis se estimaba en 224 especies, de las cuales 71 tienen distribución en el océano Atlántico (Baba et al. 2008). Recientemente, se han descrito nuevas especies de este género como en el caso de Munidopsis sarissa Lin, Osawa \& Chan, 2007, en aguas taiwanesas y Munidopsis shulerae Vásquez-Bader, Gracia \& Lemaitre, 2014, para el Atlántico Occidental. Los trabajos en este género han involucrado, además, análisis filogenéticos y morfológicos que han reevaluado la clasificación de muchas especies dentro de Munidopsis y contribuido a la descripción de nuevas especies sobre la base de evidencia molecular (Cubelio et al. 2007a; Jones \& Macpherson, 2007; Macpherson, 2007) y la ampliación de distribución de algunas especies (Schnael \& Bruce, 2006; Cubelio et al. 2007b; Hendrickx, 2007).

Recientemente, en el Caribe colombiano el interés por los mares profundos también ha incrementado el número de especies de la superfamilia Galatheoidea registradas. En el año 2008, el número de especies de la superfamilia Galatheoidea era de 43 y estaban asignadas a ocho géneros (Navas et al. 2003; Fierro et al. 2008). En recientes trabajos, se han registrado otras dos especies del género: Munidopsis robusta Milne-Edwards, 1880, y Munidopsis cubensis Chace, 
1942 (Ortega-Echeverría et al. 2013; Herrera-Medina et al. 2014). Este material nuevo fue recolectado durante las expediciones lideradas por el Instituto de Investigaciones Marinas y Costeras "José Benito Vives deAndreis" -INVEMAR- durante los muestreos de los bloques de exploración petrolera, en el Caribe colombiano, y depositado en el Museo de Historia Natural Marina de Colombia. Investigaciones similares realizadas por el proyecto "Caracterización ambiental en el área de interés dentro del bloque Guajira Offshore \# 3, Caribe colombiano", permitieron la recolección de ejemplares de Munidopsis bermudezi Chace, 1939, siendo este el primer registro de la especie para el mar Caribe colombiano.

\section{MATERIALES Y MÉTODOS}

El bloque Guajira Offshore \# 3 está ubicado frente a la costa noroccidental de la península de la Guajira. En su punto más cercano a la costa, el bloque se encuentra a 69 km. Posee un área de 949350 ha. La profundidad en esta zona oscila entre 1 500-4 000 m (com. escrita SHELL, 19 de septiembre de 2012).

El muestreo se realizó sobre una grilla de 14 estaciones. En cada una de ellas se desplegó un tren de 10 nasas durante un periodo de 24 horas. Este arte de pesca está formado por una estructura con forma cúbica de $1 \mathrm{mx} 1$ $\mathrm{m} \times 0.35 \mathrm{~m}$, moldeada por una varilla de hierro de $1 / 2$ " de espesor, la cual estaba forrada con una red de nailon negra con nudo y ojo de 1", a excepción de la base, a la que se cubre con una malla plástica con ojo de $1 / 2$ ". Posee a un lado una entrada en forma cónica de ingreso fácil para los organismos, pero de baja probabilidad de escape. Se utilizaron perlas luminosas y una bolsa con carnada como estímulos físicos y químicos, respectivamente, para atraer a los organismos presentes en el lugar de muestreo. En cada estación fue calado un tren (grupos de varias nasas conectadas a una línea principal) de 10 nasas separadas $50 \mathrm{~m}$ una de la otra y unidas a la superficie por un cabo de $3000 \mathrm{~m}$. En el Cuadro 1 se incluye la posición geográfica de las estaciones donde se recolectaron los ejemplares de M. bermudezi.

Los ejemplares se identificaron utilizando claves taxonómicas de Pequegnat y Pequegnat $(1970 ; 1971)$, confirmando la identificación con la descripción original de la especie de acuerdo con el trabajo publicado por Chace (1939). Se incluyeron datos sobre Longitud del Caparazón (lc), Ancho del Caparazón (ac) en milímetros (mm) y género de los individuos observados.

Cuadro 1. Coordenadas de las estaciones donde se recolectaron los ejemplares de Munidopsis bermudezi Chace, 1939

Table 1. Coordinates of the stations where the Munidopsis bermudezi Chace, 1939 specimens were collected

\begin{tabular}{ccc}
\hline Estaciones & Latitud & Longitud \\
\hline E IIa & $12^{\circ} 43^{\prime} 37^{\prime \prime}$ & $73^{\circ} 10^{\prime} 41^{\prime \prime}$ \\
E III & $12^{\circ} 42^{\prime} 32^{\prime \prime}$ & $7^{\circ} 07^{\prime} 03^{\prime \prime}$ \\
E IV & $12^{\circ} 39^{\prime} 48^{\prime \prime}$ & $73^{\circ} 10^{\prime} 55^{\prime \prime}$ \\
\hline
\end{tabular}




\section{RESULTADOS Y DISCUSIÓN}

Munidopsis bermudezi Chace, 1939

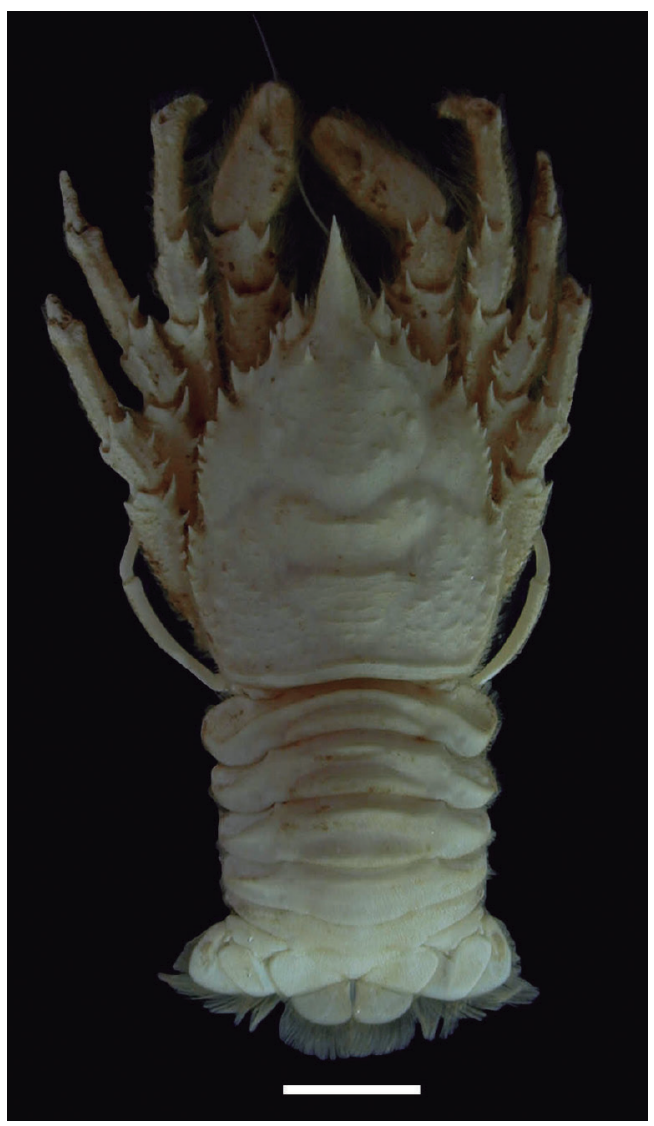

Fig. 1. Munidopsis bermudezi Chace, 1939. Vista dorsal. Hembra lc 22.82 ac $20.81 \mathrm{~mm}$, Est. IV. Profundidad $2717-2724 \mathrm{~m}$. Escala $1 \mathrm{~cm}$

Fig. 1. Munidopsis bermudezi Chace, 1939. Dorsal view. Female: cl 22.82 mm, cw $20.81 \mathrm{~mm}$. Est. IV. Depth $2717-2724 \mathrm{~m}$. Scale $1 \mathrm{~cm}$

Referencias: Chace, 1939; Chace, 1942; Pequegnat y Pequegnat, 1970; Laird et al. 1976; Gore, 1983; Baba, 2005; Baba et al. 2008; Fierro et al. 2008.
Material recolectado: Hembra lc 22.82 ac $20.81 \mathrm{~mm}$, Est. IV. Profundidad 2 717-2 $724 \mathrm{~m}$. Macho lc 22.24 ac $17.67 \mathrm{~mm}$, Est. IIa. Profundidad 2 718-2 $720 \mathrm{~m}$. Macho LC 16.09 ac $14.55 \mathrm{~mm}$, Est. III. Profundidad 2 723-2 $727 \mathrm{~m}$.

\section{Diagnosis}

Caparazón del cefalotórax ligeramente más largo que ancho, pubescente sobre toda la superficie. Presenta una espina postantenal fuerte sobre el borde lateral del caparazón, seguida de una anterolateral, separada de la primera por una emarginación semicircular. Después de las espinas anterolaterales, presenta una tercera espina conspicua seguida por cuatro o cinco espinas más reducidas, disminuyendo en tamaño gradualmente hacia la parte posterior. Detrás de la rama posterior del surco cervical, el caparazón se vuelve más ancho, presentando una espina anterior prominente en la región branquial posterior. Detrás de esta espina, el borde del caparazón está armado con dos o tres espinas pequeñas. El resto de la superficie del caparazón no presenta espinas, excepto el par de espinas postrostrales sobre la región gástrica anterior. Rostro ligeramente triangular, más corto que la longitud del caparazón, sin carinas ni surcos en la línea media dorsal. Abdomen pubescente. Los somites segundo a cuarto bicarinados dorsalmente, la carina anterior extendiéndose casi hasta el margen de la pleura y la posterior limita el tergito. Pedúnculos 
oculares fijos armados con una fuerte espina dorsal distal en la base, córnea incolora. Mero del tercer par de maxilípedos armados en el margen interno con tres espinas puntiagudas. Pereiópodos caminadores pubescentes y quelípedos más cortos que el caparazón (incluyendo el rostro), con una espina en el isquión y una línea de seis espinas dorsales sobre el mero y dos espinas distales; carpo con cinco espinas dorsales y una más pequeña en el margen de la quela, cerca a la base del dáctilo. Quela corta y ancha, con los dedos fijo y móvil tan largos como la palma (Chace, 1939).

\section{Observaciones}

Una de las especies más cercanamente relacionadas con Munidopsis bermudezi es M. teretis Baba, 2005, descrita con ejemplares recolectados en el mar de Tasmania, ubicado al suroeste del océano Pacífico (Baba, 2005). Las principales diferencias entre ambas especies radican en la presencia en $M$. bermudezi de un par de espinas gástricas conspicuas, mientras que en $M$. teretis son tubérculos, la presencia de un proceso agudo en la región branquial en $M$. teretis ausente en $M$. bermudezi y la ausencia de una carina en la mitad posterior del caparazón de $M$. bermudezi. Otra de las especies morfológicamente relacionadas es M. ceratophthalma Alcock, 1901, recolectada en aguas del mar de Andaman, sugiriendo un posible escenario de especiación para este grupo en el océano de Tetis, durante el Mesozoico, tal y como lo indica Navas (2011).

\section{Hábitat}

Los especímenes se capturaron en la masa de Agua Profunda del Atlántico Norte (North Atlantic Deep Water - NADW), presente en el Caribe por debajo de los $1000 \mathrm{~m}$ con temperaturas cercanas a $\operatorname{los} 4^{\circ} \mathrm{C}$ (Emery \& Meincke, 1986; Giraldo, 1994). A la profundidad de captura se presentó una temperatura de $4.1^{\circ} \mathrm{C}$, salinidad de 35 en promedio, saturación de $\mathrm{O}_{2}$ de $6.7 \%$ y de 8.0 PSU. El sedimento estaba conformado principalmente por limos con porcentajes superiores a $98 \%$, con un porcentaje de carbonato entre 9.39 y $15.48 \%$ y de 1.7 y $8.8 \%$ de materia orgánica.

\section{Distribución y ámbito batimétrico}

M. bermudezi es una especie con una distribución amplia en el océano Atlántico (Gore, 1983), habiéndose publicado registros en la cuenca venezolana (Gore, 1983), frente a las costas de Virginia (Laird et al. 1976), de Cuba (Chace, 1939; 1942), en el golfo de México (Pequegnat \& Pequegnat, 1970) y en el Atlántico central (Wenner, 1982), por lo que la presencia de esta especie en el Caribe colombiano era de esperarse. Sin embargo, dada su preferencia por profundidades que superan los $2000 \mathrm{~m}$ (Macpherson \& Segonzac, 2005), su captura no es frecuente. En el Caribe colombiano, los ejemplares fueron recolectados en la Ecorregión Oceánica del Caribe, a profundidades entre 2717 y $2727 \mathrm{~m}$, a $147 \mathrm{~km}$ de distancia frente a la costa de la vereda La Enea, corregimiento de Camarones, en el departamento de la Guajira, lo que 
confirma el hábito de las especies del género como especies bentónicas de fondos blandos del talud continental.

\section{AGRADECIMINETOS}

Expresamos nuestros agradecimientos al INVEMAR por facilitarnos la información ambiental y el material recolectado en el marco del proyecto: Caracterización ambiental en el área de interés dentro del bloque Guajira Offshore \# 3, Caribe colombiano. Contribución No. 439 del Instituto de Estudios en Ciencias del Mar, CECIMAR, Sede Caribe, Universidad Nacional de Colombia. Contribución No. CRTB1155 del Instituto de Investigaciones Marinas y Costeras, INVEMAR. Y los evaluadores por sus comentarios.

\section{BIBLIOGRAFÍA}

Ahyong, S. T., Baba, K., Macpherson, E. \& Poore, G. C. B. (2010). A new classification of the Galatheoidea (Crustacea: Decapoda: Anomura). Zootaxa, 2676, 57-68.

Alcock, A. (1901). A descriptive catalogue of the Indian Deep-Sea Crustacea, Decapoda Macrura and Anomala, in the Indian Museum. Being a revised account of the deepsea species collected by the Royal Indian Marine Survey Ship "Investigator". Calcuta, India: Price Ten Rupees. http://dx.doi.org/10.5962/ bhl.title.30840

Baba, K. (2005). Deep-sea chirostylid and galatheid crustaceans (Decapoda: Anomura) from the Indo-Pacific, with a list of species. En T. Wolff
(Ed.), Galathea Report. Scientific results of the Danish deep-sea expedition round the World 1950-52, 20 (pp. 1-317). Stenstrup, Denmark: Apollo Books.

Baba, K., Macpherson, E., Poore, G. C. B., Ahyong, S. T., Bermúdez, A., Cabezas, P., Lin, C. W., Nizinski, M. S., Rodrigues, C. \& Schnael, K. E. (2008). Catalogue of squat lobsters of the world (Crustacea: Decapoda: Anomura-families Chirostylidae, Galatheidae and Kiwaidae). Zootaxa, 1905, 1-220.

Cubelio, S. S., Tsuchida, S., Hendrickx, M. E., Kado, R. \& Watanabe, S. (2007a). A new species of vent associated Munidopsis (Crustacea: Decapoda: Anomura: Galatheidae) from the Western Pacific, with notes on its genetic identification. Zootaxa, 1435, 25-36.

Cubelio, S. S., Tsuchida, S. \& Watanabe, S. (2007b). Vent associated $M u$ nidopsis (Decapoda: Anomura: Galatheidae) from Brothers Seamount, Kermadec arc, southwest Pacific, with description of one new species. J. Crustacean Biol., 27(3), 513-519. http://dx.doi.org/10.1651/02780372(2007)27[513:VAMDAG] 2.0.CO;2

Chace, F. A. Jr. (1939). Reports on the scientific results of the first Atlantis Expedition to the West Indies, under the joint auspices of the University of Havana and Harvard University: Preliminary descriptions of one new genus and seventeen new species of decapod and stomatopod 
Crustacea. Mem. Soc. Cubana Hist. Nat., 13(1), 31-54.

Chace, F. A. Jr. (1942). Reports on the scientific results of the Atlantis Expeditions to the West Indies, under the joint auspices of the University of Havana and Harvard University The anomuran crustacean I Galatheidea. Torreia, Havana, 11, 1-106.

Emery, W. J. \& Meincke, J. (1986). Global water masses: summary and review. Oceanogl. Acta, 9, 383-391. Escobar-Briones, E. (2014). ¿Qué es el mar profundo? En A. Low-Pfeng \& M. Peters-Recagno (Eds.), La Frontera Final: El Océano Profundo (pp. 1-10). México: Secretaría de Medio Ambiente y Recursos Naturales.

Fierro, M., Navas, G. R., Bermúdez, A. \& Campos, N. H. (2008). Lista de chequeo de las familias Galatheidae y Chirostylidae (Crustacea: Decapoda: Anomura) del Neotrópico. Biota Colombiana, 9(1), 1-20.

Giraldo, L. O. (1994). Análisis de masas de agua y control de calidad de la información oceanográfica. B. Cientifico Cent. Invest. Oceanográficas e Hidrográficas Caribe CIOH, 15, 17-38. Gore, R. H. (1983). Notes on rare species of Munidopsis (Anomura: Galatheidae) and Ethusina (Brachyura: Dorippidae) collected by the USNS Barlett in the Venezuela Basin, Caribbean Sea. Proc. Aca. Nat. Sci. Philadelphia, 135, 200-217.

Hendrickx, M. E. (2007). Specimens of Munidopsis (Decapoda, Anomura, Galatheidae) collected by the $\mathrm{R} / \mathrm{V}$
"Revelle" (May 2004) in the Gulf of California, Mexico. Crustaceana, 80(5), 597-601. http://dx.doi. org/10.1163/156854007780765551

Herrera-Medina, Y., Bermúdez, A. \& Navas, G. R. (2014). Primer registro de Munidopsis cubensis (Crustacea: Anomura: Munidopsidae) en el mar Caribe Sur de Colombia. Rev. Acad. Colombiana Cienc. Exactas, Físicas y Naturales, 37(146), 88-92. http:// dx.doi.org/10.18257/raccefyn.42

Jones, J. \& Macpherson, E. (2007). Molecular phylogeny of the East Pacific squat lobsters of the genus Munidopsis (Decapoda: Galatheidae) with the descriptions of seven new species. J. Crustacean Biol., 27(3), 477-501. http://dx.doi. org/10.1651/S-2791.1

Laird, C., Lewis, E. G. \& Haefner, P. A. Jr. (1976). Occurrence of two galatheid crustaceans, Munida forceps and $\mathrm{Mu}$ nidopsis bermudezi, in the Chesapeake Bight of the western north Atlantic Ocean. Fish. Bull., 74(2), 462-463.

Lin, C. W., Osawa, M. \& Chan, T. Y. (2007). A new Munidopsis (Crustacea: Decapoda: Galatheidae) associated with gorgonian corals from the deep waters off Taiwan. Proc. Biol. Soc. Washington, 120(2), 167-174. http:// dx.doi.org/10.2988/0006-324X(2007) 120[167:ANMCDG]2.0.CO;2

Macpherson, E. (2007). Species of the genus Munidopsis Whiteaves, 1784 from the Indian and Pacific Oceans and reestablishment of the genus Galacantha A. MilneEdwards, 1880 (Crustacea, Decapoda, Galatheidae). Zootaxa, 1417, 1-135.

Macpherson, E. \& Segonzac, M. (2005). Species of the genus Munidopsis 
(Crustacea, Decapoda, Galatheidae) from the deep Atlantic Ocean, including cold-seep and hydrothermal vent areas. Zootaxa, 1095, 1-60.

Navas, G. R. (2011). Taxonomía, distribución y posibles eventos de especiación y dispersión de los crustáceos galateideos (Decapoda: Anomura) presentes en aguas colombianas. Tesis de Doctorado no publicada, Universidad Nacional de Colombia, Santa Marta.

Navas, G. R., Bermúdez, A., Cruz, N. \& Campos, N. H. (2003). Galateideos (Decapoda, Anomura, Galatheidae) del Caribe colombiano, incluyendo doce primeros registros. B. Investig. Mar. Cos., 32, 3-18.

Ortega-Echeverría, C., Bermúdez, A. \& Navas, G. R. (2013). Presencia de Munidopsis robusta en el Caribe colombiano (A. Milne Edwards, 1880) (Crustacea: Anomura: Munidopsidae). Universitas Scientiarum, 19(1), 43-49. http://dx.doi.org/10.11144/Javeriana. SC19-1.pmrc

Ortmann,A.E.(1898). Crustacea, Malacostraca.

En A. Gerstäcker \& A. E. Ortmann (Eds.), Die Klassen und Ordnungen der Arthropoden wissenschaftlich dargestellt in Wort und Bild, in H.G. Bronn's Die Klassen und Ordnungen der Thier-Reichs wissenschaftlich dargestellt in Wortund Bild (pp. 1057-1168). Leipzig, Germany: Winter'sche Verlagshandlung, C. F.

Pequegnat, L. H. \& Pequegnat, W. E. (1970). Deep sea anomurans of superfamily Galatheoidea with descriptions of three new species. En W. E. Pequegnat \& F. A. Chace, Jr. (Eds.), Contributions on the biology of the Gulf of Mexico (pp. 125-170). Houston, EE. UU.: Gulf Publishing Co.

Pequegnat, W. E. \& Pequegnat, L. H. (1971). New species and new records of Munidopsis (Decapoda: Galatheidae) from the Gulf of Mexico and Caribbean Sea. Houston, Texas: Texas A\&M University Oceanogr. Stud., Gulf Publ. Co., 1, 1-24.

Schnael, K. E. \& Bruce, N. L. (2006). New records of Munidopsis (Crustacea: Anomura: Galatheidae) from New Zealand with description of two new species from a seamount and underwater canyon. Zootaxa, 1172, 49-67.

Vásquez-Bader, A. R., Gracia, A. \& Lemaitre, R. (2014). A new species of Munidopsis Whiteaves, 1874 (Crustacea: Anomura: Galatheoidea: Munidopsidae) from the Gulf of Mexico and Caribbean. Zootaxa, 3821(3), 354-362. http://dx.doi.org/10.11646/ zootaxa.3821.3.4

Wenner, E. L. (1982). Notes on the distribution and biology of Galatheidae and Chirostylidae (Decapoda: Anomura) from the middle Atlantic Bight. $J$. Crustacean Biol., 2(3), 360-377. http:// dx.doi.org/10.2307/1548053

Whiteaves, J. F. (1874). On recent deepsea dredging operations in the Gulf of St. Lawrence. Amer. J. Sci., Ser., 3, 7, 210-219. http://dx.doi.org/10.2475/ ajs.s3-7.39.210 